(c) American Dairy Science Association, 2004.

\title{
Derivation and Calculation of Approximate Reliabilities and Daughter Yield-Deviations of a Random Regression Test-Day Model for Genetic Evaluation of Dairy Cattle
}

\author{
Z. Liu, F. Reinhardt, A. Bünger, and R. Reents \\ VIT, Heideweg 1, D-27283 Verden, Germany
}

\section{INTRODUCTION}

Genetic evaluation of dairy cattle using test-day yields or SCS, instead of 305-d lactation records, has become a common practice in many countries (Reents et al., 1995; Strandén and Lidauer, 1999; Schaeffer et al., 2000; Haile-Mariam et al., 2001; De Roos et al., 2002; Wiggans et al., 2002). Since May 2003, a random regression test-day model (RRTDM) has been implemented for joint production-trait evaluations for Holstein, Red, and Jersey cattle from Austria, Germany, and Luxembourg (Liu et al., 2001). This evaluation includes over 10 million cows with test-day records and over 250 million equations for each trait. For the purpose of approximating reliabilities of EBV in large-scale national genetic evaluations, the daughter-equivalents (DE) concept (VanRaden and Wiggans, 1991) was shown to be accurate and efficient. In recent years, several new reliability methods were developed, e.g., information source method (Harris and Johnson, 1998) and effective number of progeny (ENP) method (Koots et al., 1997; Jamrozik et al., 2000). Reliability approximation was also extended to multiple-trait models (Graser and Tier, 1997; Strabel et al., 2001; VanRaden, 2001). Fikse and Banos (2001) applied the reliability method of Harris and Johnson (1998) in a study on alternative weighting factors for international bull evaluation, which resulted in the development of the effective daughter contribution (EDC) method for computing reliability associated with daughter information of bulls. All of the methods are closely related to the DE method. Although EDC and DE have been widely used for single-trait models, their extension to multipletrait models, including RRTDM, is limited. The multitrait extensions have typically combined information from multiple traits at an early stage, which may lead to loss of important information.

Daughter yield deviations (DYD) of a bull are, by definition, the average of the daughters' performance adjusted for fixed and nongenetic random effects of the daughters and genetic effects of the bull's mates. Daughter yield deviations are not regressed and are a more independent measure of phenotypic performance of a bull's daughters than EBV (VanRaden and Wig- 
gans, 1991). Thanks to this property, DYD are widely used in genetic analyses, such as in mapping quantitative trait loci (Szyda et al., 2002), validating national genetic trends (Boichard et al., 1995) and a 2-step multiple-trait genetic evaluation (Ducrocq et al., 2001). For repeatability animal models applied to lactation records, VanRaden and Wiggans (1991) showed the calculation of DYD. Mrode and Swanson (2002) extended the method to an RRTDM; however, computation of reliability associated with DYD, estimable lactation of DYD, and short lactation problems were not addressed.

The objectives of this study were (1) to extend the single-trait DE concept to multiple-trait models for approximating the reliability of EBV, and (2) to develop a method for calculating DYD under general multipletrait models and to verify the DYD method.

\section{MATERIALS AND METHODS}

\section{Theory of the Multiple-Trait EDC Method}

The general idea behind DE, EDC, or ENP methods is to express the amount of information contributed by different sources for an animal in the number of standard daughters (progeny) that would give equal reliability by treating the animal as a standard sire. A standard sire is defined as an animal without its own performance records and having both parents missing, and only his daughters (progeny) contribute to the estimation of his breeding values. Further, it is assumed that the whole population is comprised of a single halfsib family, and all daughters of the sire have their dams missing. Under the above assumptions, EBV or their reliabilities of the standard sire under a sire model and an animal model are equal. A multiple-trait animal model in a general form

$$
\mathbf{y}=\mathbf{X b}+\mathbf{Z}_{p} \mathbf{p}+\mathbf{Z a}+\mathbf{e}
$$

can be expressed as a multiple-trait sire model for EBV of the sire

$$
\mathbf{y}=\mathbf{X b}+\mathbf{Z}_{p} \mathbf{p}+\mathbf{Z}_{s} \mathbf{a}_{s}+\mathbf{e}_{s},
$$

where $\mathbf{y}$ is a vector of records of a daughter; $\mathbf{X}, \mathbf{Z}_{p}$, and $\mathbf{Z}$ are design matrices for all fixed effects $(\mathbf{b})$; all nongenetic random effects (p); and additive genetic effects (a), respectively. Variance and covariance matrices are $\mathbf{G}_{0}, \mathbf{P}_{0}$, and $\mathbf{R}_{0}$ for the genetic, nongenetic random, and error effects, respectively. It is assumed that these random effects are not correlated with each other. Design matrix $\mathbf{Z}_{s}$ is for genetic effects of the sire. (Co)variance matrices for the sire effects $\left(\mathbf{a}_{s}\right)$ and residual $\operatorname{effect}\left(\mathbf{e}_{s}\right)$ of the sire model [2] $\operatorname{are} \operatorname{var}\left(\mathbf{a}_{s}\right)=\mathbf{G}_{s}=\mathbf{1} / \mathbf{4} \mathbf{G}_{0}$ and $\operatorname{var}\left(\mathbf{e}_{s}\right)=\mathbf{3} / \mathbf{4} \mathbf{G}_{0}+\mathbf{R}_{0}$. For estimating the sire's breeding values, all fixed and nongenetic random effects of the daughters are absorbed into their genetic effects that are further absorbed into the genetic effects of the sire. The absorptions result in mixed model equations

$$
\left(\boldsymbol{\Psi}+\mathbf{G}_{s}^{-1}\right) \hat{\mathbf{a}}_{s}=\Delta,
$$

where $\Psi$ and $\Delta$ are the part of the left-hand side and right-hand side of the equations after the absorptions, respectively. Let $\mathbf{C}=\left(\boldsymbol{\Psi}+\mathbf{G}_{s}^{-1}\right)^{-1}$, then a matrix $\Re$ can be defined as

$$
\begin{gathered}
\Re=\mathbf{G}_{s}^{-1}\left(\mathbf{G}_{s}-\mathbf{C}\right)=\mathbf{I}-\mathbf{G}_{s}^{-1} \mathbf{C}=\mathbf{I} \\
-\mathbf{G}_{s}^{-1}\left(\boldsymbol{\Psi}+\mathbf{G}_{s}^{-1}\right)^{-1}=\mathbf{I}-\left(\boldsymbol{\Psi} \mathbf{G}_{s}+\mathbf{I}\right)^{-1}
\end{gathered}
$$

The matrix $\Re$ can be converted back to an EDC matrix $(\Psi)$ with

$$
\boldsymbol{\Psi}=\left[(\mathbf{I}-\mathfrak{R})^{-1}-\mathbf{I}\right] \mathbf{G}_{s}^{-1}
$$

The matrix $\Re$ will be referred to as a reliability matrix. Although it does not contain reliabilities, it can be used to derive reliabilities for all traits, and can be viewed as a multivariate generalization of the scalar reliability $\left(R^{2}\right)$ of single-trait models. Matrix $\Re$ is equal to $R^{2}$ in a single-trait case, as shown from Formula [4]:

$$
\begin{aligned}
& \Re=\mathbf{I}-\left(\boldsymbol{\Psi} \mathbf{G}_{s}+\mathbf{I}\right)^{-1}=1-\left(n \sigma_{e}^{-2} \sigma_{s}^{2}+1\right)^{-1}=1 \\
&-(n / k+1)^{-1}=n /(n+k)=R^{2},
\end{aligned}
$$

where $k=\sigma_{e}^{2} / \sigma_{s}^{2}$, and $\sigma_{s}^{2}$ and $\sigma_{e}^{2}$ are sire and residual variances of a single-trait sire model, respectively. Similarly, the matrix $\Psi$ provides the equivalent to the effective number of daughters $(n)$ of single-trait models

$$
n=k R^{2} /\left(1-R^{2}\right),
$$

which can be obtained by simplifying formula [5] to

$$
n \sigma_{s}^{-2}=\left(1 /\left(1-R^{2}\right)-1\right) \sigma_{s}^{-2} .
$$

The reliability and EDC matrices constitute the core of the multiple trait effective daughter contribution (MTEDC) method for approximating reliabilities under general multiple-trait models. The reliability matrix $R$ is asymmetric, and it usually has nonzero off-diagonal elements. $\Re$ has a row of zeros corresponding to a missing trait. In contrast to $\Re$, the EDC matrix $\Psi$ is symmetric, and it normally has nonzero off-diagonal elements. For a missing trait, $\Psi$ has a row and a column of zeros. 
The EDC matrices from different information sources of the same animal can be summed up together. It can be proved using formulas [4] and [5] that a symmetric EDC matrix is always guaranteed by converting a nonsymmetric reliability matrix. Additionally, the inverse of matrix $\mathbf{I}-\mathfrak{R}$ in formula [5], which equals $\mathbf{C}^{-1} \mathbf{G}_{s}$, exists under all conditions. The multitrait reliability matrix $\Re$ does not contain final reliability values of EBV, unless the traits are not correlated. By defining appropriate vectors $\mathbf{v}_{i}$ and $\mathbf{v}_{j}$, final reliability or coreliability of any linear function of EBV can be calculated with

$$
R_{i j}^{2}=\mathbf{v}_{i}^{\prime} \mathbf{G}_{0} \Re \mathbf{v}_{j} / \mathbf{v}_{i}^{\prime} \mathbf{G}_{0} \mathbf{v}_{j}
$$

Approximating reliabilities for an RRTDM. By extending the daughter-equivalents method (VanRaden and Wiggans, 1991) of single-trait models, reliability approximation using the MTEDC was developed under general multiple-trait models. The procedure of reliability approximation using MTEDC is illustrated below with an RRTDM (Liu et al., 2001):

$$
\mathbf{y}=\mathbf{X}_{h} \mathbf{h}+\mathbf{X}_{f} \mathbf{f}+\mathbf{Z}_{p} \mathbf{p}+\mathbf{Z} \mathbf{a}+\mathbf{e},
$$

where $\mathbf{h}$ represents fixed effects of herd test-date-parity milking frequency, $\mathbf{f}$ is the effects of fixed lactation curves, and $\mathbf{p}$ is the permanent environmental (p.e.) effects, and $\mathbf{Z}_{h}$ and $\mathbf{X}_{f}$ are design matrices for $\mathbf{h}$ and f, respectively.

Reliability approximation using the MTEDC method consists of 3 steps in the sequence-computing contributions by own data, progeny, and parental average. Computing the data contribution for a cow involves absorbing all fixed effects and the nongenetic random effects into her genetic effects. By absorbing the major fixed herd test-date-parity milking frequency effect and ignoring the minor effect of fixed lactation curves (Liu et al., 2001), the left-hand side of the mixed model equations pertinent to a cow $i$ with records becomes

$$
\left[\begin{array}{cc}
\mathbf{Z}_{p}^{\prime} \mathbf{M} \mathbf{Z}_{p}+\mathbf{P}_{0}^{-1} & \mathbf{Z}_{p}^{\prime} \mathbf{M} \mathbf{Z} \\
\mathbf{Z}^{\prime} \mathbf{M} \mathbf{Z}_{p} & \mathbf{Z}^{\prime} \mathbf{M Z}+a^{i i} \mathbf{G}_{0}^{-1}
\end{array}\right],
$$

where $\mathbf{M}=\mathbf{R}^{-1}-\mathbf{R}^{-1} \mathbf{X}_{h}\left(\mathbf{X}_{h}^{\prime} \mathbf{R}^{-1} \mathbf{X}_{h}\right)^{-} \mathbf{X}_{h}^{\prime} \mathbf{R}^{-1}$ and $\mathbf{R}$ is the error (co)variance matrix for the records of the cow, and $a^{i i}$ is the diagonal element of inverse of the relationship matrix for the cow. Further absorption of her p.e. effects gives $\left[\mathbf{Z}^{\prime} \mathbf{W Z}+a^{i i} \mathbf{G}_{0}^{-1}\right]$ with $\mathbf{W}=\mathbf{M}-\mathbf{M Z}_{p}\left(\mathbf{Z}_{p}^{\prime}\right.$ $\left.\mathbf{M Z} \mathbf{Z}_{p}+\mathbf{P}_{0}^{-1}\right)^{-1} \mathbf{Z}_{p}^{\prime} \mathbf{M}$. The effective information contributed by her own test-day records is summarized in matrix $\mathbf{Z} \mathbf{W Z}$, from which a reliability matrix due to own records $(\mathrm{Y})$ is computed using

$$
\Re_{\mathrm{Y}}=\mathbf{I}-\left(\mathbf{Z}^{\prime} \mathbf{W Z G}_{0}+\mathbf{I}\right)^{-1}
$$

and then the reliability matrix is converted to an EDC matrix based on Formula [5]

$$
\boldsymbol{\Psi}_{\mathrm{Y}}=4\left[\left(\mathbf{I}-\mathfrak{R}_{\mathrm{Y}}\right)^{-1}-\mathbf{I}\right] \mathbf{G}_{0}^{-1} .
$$

It can be shown that $\Psi_{\mathrm{Y}}=4 \mathbf{Z} \mathbf{W Z}$ for cows with records.

Collecting EDC from all progeny is done by processing pedigree files from the youngest to oldest animals (VanRaden and Wiggans, 1991). An animal's reliability contributed by a progeny adjusted for mate effect (P-M) is computed as

$$
\Re_{\mathrm{P}-\mathrm{M}}=\frac{1}{4}\left[\mathbf{E}-\mathbf{E}\left(\mathbf{E}+\left(\mathbf{I}-\frac{1}{4} \Re_{\mathrm{M}}^{*}\right)^{-1}\right)^{-1} \mathbf{E}\right],
$$

where $\Re_{\mathrm{M}}{ }^{*}$ is the mate's reliability calculated excluding EDC of this progeny and

$$
\mathbf{E}=\left(\mathbf{I}-\Re_{\mathrm{P}}^{*}\right)^{-1}-\mathbf{I},
$$

with $\Re_{\mathrm{P}}{ }^{*}$ being the progeny's reliability, including information from its records and its progeny but not from its parents. The reliability matrix $\Re_{\mathrm{P}-\mathrm{M}}$ is converted to an EDC matrix

$$
\boldsymbol{\Psi}_{\mathrm{P}-\mathrm{M}}=4\left[\left(\mathbf{I}-\mathfrak{R}_{\mathrm{P}-\mathrm{M}}\right)^{-1}-\mathbf{I}\right] \mathbf{G}_{0}^{-1}
$$

for the progeny. Note that $\Re_{\mathrm{P}-\mathrm{M}}=\frac{1}{4} \Re_{\mathrm{P}}$, if $\Re_{\mathrm{M}}^{*}=0$. For the derivation of formula [13], see Appendix 1.

After the EDC from all progeny has been accumulated, pedigree files are processed from the oldest to youngest animals to collect parental contribution (VanRaden and Wiggans, 1991). To avoid double counting an animal's contribution to its parents, the EDC provided by the animal to its parents must first be subtracted from its parental EDC. Then the resulting EDC $\left(\Psi^{*}\right)$ is converted to reliability using

$$
\Re^{*}=\mathbf{I}-\left(\frac{1}{4} \Psi^{*} \mathbf{G}_{0}+\mathbf{I}\right)^{-1}
$$

for computing parental contribution to the animal. Assuming that both parents are not related,

$$
\Re_{\mathrm{PA}} \approx \frac{1}{4}\left(\Re_{\text {sire }}^{*}+\mathfrak{R}_{\text {dam }}^{*}\right)
$$

and 


$$
\boldsymbol{\Psi}_{\mathrm{PA}}=4\left[\left(\mathbf{I}-\Re_{\mathrm{PA}}\right)^{-1}-\mathbf{I}\right] \mathbf{G}_{0}^{-1}
$$

can be used to compute reliability and the EDC matrix provided by parental average to the animal, respectively.

When the 3 steps have been completed, the total EDC $\left(\boldsymbol{\Psi}_{\mathrm{T}}\right)$ is calculated with

$$
\boldsymbol{\Psi}_{\mathrm{T}}=\boldsymbol{\Psi}_{\mathrm{PA}}+\boldsymbol{\Psi}_{\mathrm{Y}}+\sum \boldsymbol{\Psi}_{\mathrm{P}-\mathrm{M}}
$$

for each animal and converted to a total reliability matrix with

$$
\mathfrak{R}_{\mathrm{T}}=\mathbf{I}-\left(\frac{1}{4} \boldsymbol{\Psi}_{\mathrm{T}} \mathbf{G}_{0}+\mathbf{I}\right)^{-1}
$$

Using the total reliability matrix, the reliability value of any linear function of genetic random regression coefficient estimates can be readily derived. An example is given here to show the calculation of reliability of combined lactation EBV using the total reliability matrix $\Re_{\mathrm{T}}$. The breeding value on a 305-d lactation basis is defined as $u_{\mathrm{L}_{\mathrm{i}}}=\sum_{j}^{305} u_{\mathrm{ij}}=\sum_{j}^{305} \mathbf{t}_{j}^{\prime} \mathbf{a}_{i}=\mathbf{1}^{\prime} \mathbf{T} \mathbf{a}_{i}$, and combined lactation breeding value as $u_{c o m b}=\mathbf{w}^{\prime} \mathbf{u}_{L}=\mathbf{v} \mathbf{a}$, where $u_{L_{i}}$ is a 305-day lactation breeding value for lactation $i, \mathbf{u}_{L}$ contains lactation breeding values of the first 3 lactations, $\mathbf{t}_{j}$ contains Legendre polynomial constants (Liu et al., 2001) for $\operatorname{DIM} j, \mathbf{a}_{i}$ contains regression coefficients of genetic effects for lactation $i, \mathbf{w}$ contains economic weights of the first 3 lactations, and $\mathbf{v}^{\prime}=\mathbf{w}^{\prime}$ diag $\left\{\mathbf{1}^{\prime} \mathbf{T}, \mathbf{1}^{\prime} \mathbf{T}, \mathbf{1}^{\prime} \mathbf{T}\right\}$. Because $\operatorname{var}(\mathbf{a})=\mathbf{G}_{0}$ and $\operatorname{var}(\hat{\mathbf{a}})=\mathbf{G}_{\mathbf{0}} \Re_{\mathrm{T}}$, reliability of combined lactation EBV is calculated with

$$
R_{\hat{u}_{\text {comb }}}^{2}=\mathbf{v}^{\prime} \mathbf{G}_{0} \Re_{\mathrm{T}} \mathbf{v} / \mathbf{v}^{\prime} \mathbf{G}_{0} \mathbf{v}
$$

By setting the ith element of $\mathbf{w}$ to one and the others to zero, reliability of the $i$ th lactation $\mathrm{EBV} R_{\hat{u}_{L_{i}}}^{2}$ can be calculated using the same formula for $R_{\hat{u}_{L} \text { comb }}^{2}$.

According to the MTEDC method, the relationship among animal, parents, and progeny is modeled via reliability matrix, e.g., equation [13] for calculating an animal's reliability contributed by a progeny adjusted for its mate, and formula [15] for computing the reliability of parental average. The relationships among different information sources of the same animal are modeled via EDC matrix $\Psi$, which can be summed up over information sources.

\section{Calculating DYD}

Calculation of DYD of a bull represents a process of absorbing the daughters' genetic effects using the daughters' records adjusted for all other effects and the bull's mates' breeding values. Denote $a_{*}^{i i}$ as a diagonal element of the inverse of the numerator relationship matrix corresponding to daughter $i$ of a bull, under the assumption that the daughter has no progeny, and $d_{i}$ as the diagonal element pertinent to daughter $i$ in inverse of the (co)variance matrix of Mendelian sampling $\mathbf{D}^{\mathbf{1}}$ (Mrode, 1996, p. 28). The DYD of the bull under the general multiple-trait animal model [1] is computed with

$$
\begin{gathered}
\mathbf{q}=\mathbf{B}^{-1} \xi=2\left(\sum_{i=1}^{n} d_{i} \mathbf{G}_{0}^{-1} \mathbf{Q}_{i} \mathbf{Z}_{i}^{\prime} \mathbf{R}_{i}^{-1} \mathbf{Z}_{i}\right)^{-1} \\
\sum_{i=1}^{n} d_{i} \mathbf{G}_{0}^{-1} \mathbf{Q}_{i} \mathbf{Z}_{i}^{\prime} \mathbf{R}_{i}^{-1} \\
\left(\mathbf{y}_{i}-\mathbf{X}_{i} \hat{\mathbf{b}}-\mathbf{Z}_{p i} \hat{\mathbf{p}}_{i}-\frac{1}{2} \mathbf{Z}_{i} \hat{\mathbf{a}}_{d}\right),
\end{gathered}
$$

where subscript $i$ represents daughters of the bull, $n$ is the number of daughters with records, $\hat{\mathbf{a}}_{d}$ is EBV of the dam of the daughter $i$ obtained from a complete genetic evaluation like routine national evaluation, $\mathbf{y}_{i}$ is a vector of the $i$ th daughter's test-day yields adjusted for heterogeneous herd variances, and

$$
\begin{aligned}
\mathbf{Q}_{i} & =\left(\mathbf{Z}_{i}^{\prime} \mathbf{R}_{i}^{-1} \mathbf{Z}_{i}+a_{*}^{i i} \mathbf{G}_{0}^{-1}\right)^{-1} \\
\mathbf{B} & =\sum_{i=1}^{n} \frac{1}{4} d_{i} \mathbf{G}_{0}^{-1} \mathbf{Q}_{i} \mathbf{Z}_{i}^{\prime} \mathbf{R}_{i}^{-1} \mathbf{Z}_{i} \\
\xi & =\sum_{i=1}^{n} \frac{1}{2} d_{i} \mathbf{G}_{0}^{-1} \mathbf{Q}_{i} \mathbf{Z}_{i}^{\prime}\left(\mathbf{y}_{i}-\mathbf{X}_{i} \hat{\mathbf{b}}-\mathbf{Z}_{p i} \hat{\mathbf{p}}_{i}-\frac{1}{2} \mathbf{Z}_{i} \hat{\mathbf{a}}_{d}\right)
\end{aligned}
$$

See Appendix 2 for the derivation of the DYD formula [19] under multiple-trait models.

Formula [19] for calculating DYD is equivalent to the one that VanRaden and Wiggans (1991) developed for the repeatability animal model and also similar to the one by Mrode and Swanson (2002) for RRTDM. For repeatability animal models, the DYD vector $\mathbf{q}$ becomes a scalar that is a weighted average DYD over all lactation records. For multiple lactation/trait models, q consists of DYD for each lactation/trait. As genetic effects are modeled in regression coefficients on DIM in RRTDM, $\mathbf{q}$ is also expressed in the form of regression coefficients (Mrode and Swanson, 2002). With those regression coefficients, any linear function of the DYD regression coefficients can be calculated. For RRTDM, the inverse of matrix $\mathbf{B}$ does not always exist. For instance, when all daughters of a bull have fewer testday records in a given lactation than the order of fit for genetic effects, DYD are not estimable for the bull. 
However, when at least one daughter has at least as many test-day records in a lactation as the order of fit for genetic effects, DYD of the bull are defined for this lactation. Lactation yield deviations for cows (see Appendix 2) are defined when there are at least so many test-day records available for a lactation as the order of fit for genetic effects. In case of estimable DYD, all data from daughters with defined, as well as daughters with undefined, lactation yield deviations can be used. Because of using adjusted records to derive DYD, the size of contemporary groups is not accounted for. To obtain DYD that correspond with EBV, records without contemporaries must be excluded from the DYD calculation. In case of unknown mates, $\hat{\mathbf{a}}_{d}$ is replaced with solutions of corresponding genetic groups. The DYD formula [19] for bulls and formula [B3] in Appendix 2 for average yield deviations of cows show how information on test-day level should be summarized to lactation level for test-day models in national and international genetic evaluations.

In contrast to the derivations by VanRaden and Wiggans (1991) and Mrode and Swanson (2002), the DYD formula [19] was derived from bull equations (see Appendix 2) but not from cow equations. Additionally, bulls and their mates can be inbred. Factor 2 in formula [19] reflects the fact that the derived DYD are expressed on an animal basis and not on a sire basis, as in VanRaden and Wiggans (1991) and Mrode and Swanson (2002). Formula [19] always gives zero DYD values for missing lactations/traits. The DYD Formula [19] developed for general multiple-trait models is applicable for any RRTDM and single-trait models.

Fixed effects, nongenetic random effects, and EBV of mates are subtracted from daughters' yields, but are not absorbed into the daughters' genetic effects. In DYD calculation, no reliability associated with DYD can be derived from the DYD formula [19]. However, the MTEDC can be implemented to compute reliability associated with DYD. The reliability calculation procedure for DYD using the MTEDC differs with the normal procedure of reliability approximation in that only the first 2 steps of the MTEDC are needed: calculation of data contribution for daughters with records and collecting EDC of daughters adjusted for mates' contribution. The summing of the EDC of all daughters gives EDC associated with bull's DYD q

$$
\Psi_{\mathbf{q}}=\sum \Psi_{\mathrm{P}-\mathrm{M}},
$$

with $\boldsymbol{\Psi}_{\mathrm{P}-\mathrm{M}}$ being the EDC matrix contributed by a daughter adjusted for her dam's contribution. Reliability matrix associated with $\mathbf{q}$ is computed with

$$
\Re_{\mathbf{q}}=\mathbf{I}-\left(\frac{1}{4} \boldsymbol{\Psi}_{\mathbf{q}} \mathbf{G}_{0}+\mathbf{I}\right)^{-1} .
$$

\section{Data}

Table 1 describes the data set used in the official May 2003 genetic evaluation of production traits for Austrian, German, and Luxembourg dairy cattle (includes Holstein, Red, and Jersey). The presented DYD formula [19] and associated reliability formula [21] were applied to the data set. Besides DYD of bulls, lactation yield deviations were computed for cows with records in the application.

\section{A Verification Study on the DYD Calculation and Reliability Approximation Methods}

The rationale of the verification study is that EBV of a bull obtained from DYD and associated reliabilities must be equal to those obtained from a complete mixed model equation system, including equations of genetic and nongenetic effects of the bull, his daughters, and mates. Also, approximated reliability values of the bull's EBV must be equal to his true reliability value, derived from the exact inverse of the mixed model equations.

Pedigree and data. A single half-sib family structure was assumed in the verification study. As parental contribution is irrelevant for validating the DYD formula and its associated reliability method, both parents of the bull were assumed to be unknown. All daughters of the bull had a known dam and performance records. All mates of the bull had both parents missing and full performance information, i.e., 3 complete lactations with 10 test-day records each. Each mate had only one progeny, namely a daughter of the bull. Between daughter-dam pairs, there was no additional genetic connection except the bull. With permanent environmental effects included in the study, correctness of the method of adjusting records for other effects and accuracy of the reliability calculation method could be examined; therefore, no fixed effects were included in the simulation. A monthly testing scheme was assumed to generate test-day records for daughters and dams, with first tests done at 10 DIM for mates or first-crop daughters and 15 DIM for second-crop daughters. A new test-day record was added every $30 \mathrm{~d}$ for daughters or mates.

Various scenarios were investigated for accuracy of the DYD and reliability methods. A different number of daughters was used to simulate the bull in the progeny testing program and with second-crop daughters. The daughters may have 1 to 3 lactations. Lactation progress of the daughters was simulated with a number of test-day records. Test-day yields of all mates of the simulated bull were set to zero, implying that phenotypic performance of the mates was equal to comparison-group average. All daughters' phenotypic records on all test days were simulated as deviations from their 
Table 1. Descriptive statistics of the data set for official production-trait genetic evaluation of Holstein, Red, and Jersey dairy cattle from Austria, Germany, and Luxembourg in May 2003.

\begin{tabular}{lllllll}
\hline Item & $\begin{array}{l}\text { No. of cows } \\
\text { with records }\end{array}$ & $\begin{array}{l}\text { No. of animals } \\
\text { in pedigree }\end{array}$ & $\begin{array}{l}\text { No. of } \\
\text { lactations }\end{array}$ & $\begin{array}{l}\text { No. of herd } \\
\text { test-day } \\
\text { records }\end{array}$ & $\begin{array}{l}\text { No. of test- } \\
\text { day records }\end{array}$ & $\begin{array}{l}\text { No. of equations } \\
\text { per trait }\end{array}$ \\
\hline Size & $10,683,586$ & $15,504,210$ & $21,749,808$ & $14,899,608$ & $178,824,545$ & $250,615,416$ \\
\hline
\end{tabular}

comparison groups and expressed in phenotypic standard deviations. Test-day yield deviation at a given DIM was assumed to be 0.10 of the phenotypic standard deviation of DIM for all daughters. Parameters of testday milk yield (Liu et al., 2000a) were used in generating and analyzing data.

Estimating a bull's breeding values using DYD and associated reliabilities. The solutions from the complete mixed model equation system were used in the calculation of DYD with formula [19]. For estimating the bull's breeding values using his DYD, the following mixed model equations are to be solved

$$
\begin{gathered}
\left(\boldsymbol{\Psi}_{\mathrm{b}}+\mathbf{G}_{0}^{-1}\right) \hat{\mathbf{a}}_{s}=\Delta_{\mathrm{b}} \\
\text { where } \boldsymbol{\Psi}_{\mathrm{b}}=\left[\left(\mathbf{I}-\Re_{\mathbf{q}}\right)^{-1}-\mathbf{I}\right] \mathbf{G}_{0}^{-1}
\end{gathered}
$$

and

$$
\Delta_{\mathrm{b}}=\xi+\left(\boldsymbol{\Psi}_{\mathrm{b}}-\mathbf{B}\right)\left(\mathbf{B}+\mathbf{G}_{0}^{-1}\right)^{-1} \xi
$$

The reliability matrix of the sire's EBV was calculated with $\Re_{\hat{\mathbf{a}}_{s}}=\mathbf{I}-\mathbf{G}_{0}^{-1}\left(\boldsymbol{\Psi}_{\mathrm{b}}+\mathbf{G}_{0}^{-1}\right)^{-1}$.

For international genetic evaluations, the multitrait DYD calculated with formula [19] can be used as trait values. Setting up mixed-model equations of multiple across-country evaluations based on the multitrait DYD differs with the multiple-trait bull-comparison procedure based on de-regressed proofs (Schaeffer, 2001). The least squares part of the left-hand side of the mixed-model equation corresponding to a bull with daughters having performance is $\boldsymbol{\Psi}_{\mathrm{b}}$; the right-hand side of the mixed-model equation pertinent to the bull in multitrait across-country equations is $\Delta_{\mathrm{b}}$.

\section{RESULTS AND DISCUSSION}

\section{Reliability Approximation Using the MTEDC}

The presented MTEDC method extends the single trait DE (VanRaden and Wiggans, 1991) to general multiple-trait models, including RRTDM. Therefore, it must give identical formulas as those in VanRaden and Wiggans (1991) for single-trait models, when the MTEDC is applied to single-trait models. As shown before, formulas [4] and [5] of multiple-trait models are equivalent to formulas [6] and [7] of single-trait models, respectively. Let $R_{\mathrm{p}}^{2^{*}}, R_{\mathrm{P}-\mathrm{M}}^{2}$, and $R_{\mathrm{M}}^{2 *}$ represent a progeny's reliability based on its own performance records and its descendants but not based on its parents, the animal's reliability contributed by the progeny adjusted for mate of the animal, and the reliability of the mate with EDC from this progeny excluded, respectively. Formula [13] can then be simplified to $R_{\mathrm{P}-\mathrm{M}}^{2}=\frac{R_{\mathrm{P}}^{2^{*}}}{4-R_{\mathrm{P}}^{2^{*}} R_{\mathrm{M}}^{2^{*}}}$, which is identical to $\mathrm{REL}_{\mathrm{anim}}^{*}$ derived by VanRaden and Wiggans (1991).

Using formula [13], a standard daughter can be defined. For multiple-trait models, a standard daughter is characterized as a cow having one record in all traits with an infinite number of contemporaries, and the other parent missing. This definition is an extension of Interbull's EDC definition for single-trait models but differs slightly from VanRaden and Wiggans (1991) in the assumption of the other parent. However, the difference in definitions does not have an impact on reliability because of the corresponding difference in variance ratios. For traits with repeated records, such as lactations with test-day records, one record means a complete lactation, according to MTEDC. To study the effect of the model on the value of EDC, a single trait repeatability model was applied to the lactation average SCS, and a multiple lactation RRTDM (Liu et al., 2001) was applied to test-day SCS and compared. The repeatability model was assumed to have a heritability of 0.1 and repeatability of 0.35 , whereas heritability of lactation average SCS was about 0.2 for the RRTDM (Liu et al., 2000b). Table 2 shows the EDC of an example cow in a monthly testing program during the course of lactations under both models. With only 3 tests in first lactation, the cow has 1.6 EDC for the single trait model. This means that the reliability contributed by 3 tests of her own is equal to the reliability that would be contributed by 1.6 of her standard daughters. When the cow has completed her first 3 lactations, the EDC of the repeatability model is 8.3, which is greater than the EDC of the RRTDM, which is 5.2. The phenomenon of smaller EDC of multiple-trait models than single-trait models has also been observed in Interbull's EDC for bulls of the same age from countries using different models 
Table 2. Comparison of effective daughter contribution (EDC) of a single- and multiple-trait model, illustrated with a cow in a monthly testing program.

\begin{tabular}{lll}
\hline & \multicolumn{2}{c}{ EDC of the example cow from the } \\
\cline { 2 - 3 } Own performance records of the cow & ${\text { Single-trait } \text { model }^{1}}^{1}$ & ${\text { Multiple-trait } \text { model }^{2}}^{2}$ \\
\hline Three tests from first lactation & 1.6 & 1.2 \\
Six tests from first lactation & 2.9 & 1.8 \\
First lactation completed with 10 tests & 4.3 & 2.4 \\
First two lactations completed & 6.7 & 4.1 \\
First three lactations completed & 8.3 & 5.2 \\
\hline
\end{tabular}

${ }^{1} \mathrm{~A}$ repeatability animal model applied to lactation-average SCS.

${ }^{2} \mathrm{~A}$ multiple lactation random regression test-day model applied to test-day SCS.

for national genetic evaluations. This can be explained by different definitions of standard daughter in single and multiple trait models: a standard daughter has only one complete lactation under the repeatability model but 3 complete lactations under the RRTDM.

Liu et al. (2002) compared the MTEDC and ENP (Jamrozik et al., 2000) methods for RRTDM in a simulation study. Whereas the MTEDC gave unbiased reliability estimates, ENP overestimated reliability values of bulls that had daughters with short or missing lactations. In the verification study of this research, the reliability values computed with the MTEDC were equal to true reliability values. Additionally, the MTEDC can be applied to very large populations. It has been routinely used in Germany (Liu et al., 2002) and The Netherlands (De Roos et al., 2002).

Approximating reliability using the MTEDC method does not require special handling of missing traits. In case of nonrandom distribution of relatives in contemporary groups, the loss of information must be considered in the computation of data contribution. Calculating the parental contribution to an animal requires recomputation of the animal's contribution to its parents that was done already in the calculation of prog- eny contribution, and this repetition can be avoided if the animal's contribution to its parents can be stored in the progeny contribution calculation and then be read in for the parental contribution calculation. The MTEDC makes approximations in the calculation of parental contribution by assuming that both parents are unrelated, as do all existing reliability approximation methods, to our knowledge. A problem in the calculation of progeny contribution was identified that, at the time of adjusting the contribution of a progeny to an animal for reliability of the animal's mate, the mate may not have all its progeny processed yet. This problem may be solved by using reliability values from the previous genetic evaluation as starting values for the mate; however, this bias has limited impact due to the factor 0.25 for the mate's reliability. We applied the MTEDC method to a maternal-effect model with correlated genetic effects for international genetic evaluations of calving traits. An extension of the MTEDC to nonlinear or threshold models needs to be developed for traits like herd life. Although the MTEDC has been proven to be fairly accurate for multiple-trait models, until now, in dairy cattle only, it

Table 3. Lactation daughter yield deviations (DYD) and EBV of the simulated bull, expressed in genetic standard deviations.

\begin{tabular}{|c|c|c|c|c|c|c|c|c|c|}
\hline \multirow{2}{*}{$\begin{array}{l}\text { No. of } \\
\text { daughters }\end{array}$} & \multirow{2}{*}{\multicolumn{3}{|c|}{$\begin{array}{l}\text { No. of tests } \\
\text { by lactation }\end{array}$}} & \multicolumn{2}{|c|}{ Lactation 1} & \multicolumn{2}{|c|}{ Lactation 2} & \multicolumn{2}{|c|}{ Lactation 3} \\
\hline & & & & DYD & EBV & DYD & EBV & DYD & EBV \\
\hline \multirow[t]{2}{*}{50} & 2 & 0 & 0 & & 0.23 & $\ldots$ & 0.17 & $\ldots$ & 0.18 \\
\hline & 3 & 0 & 0 & 0.52 & 0.25 & $\ldots$ & 0.19 & $\ldots$ & 0.19 \\
\hline \multirow[t]{8}{*}{100} & 3 & 0 & 0 & 0.53 & 0.27 & $\ldots$ & 0.20 & $\ldots$ & 0.21 \\
\hline & 10 & 0 & 0 & 0.34 & 0.33 & $\ldots$ & 0.27 & $\ldots$ & 0.27 \\
\hline & 10 & 2 & 0 & 0.35 & 0.34 & . & 0.35 & $\ldots$ & 0.34 \\
\hline & 10 & 3 & 0 & 0.35 & 0.34 & 0.63 & 0.35 & $\ldots$ & 0.35 \\
\hline & 10 & 10 & 0 & 0.35 & 0.34 & 0.42 & 0.40 & $\ldots$ & 0.39 \\
\hline & 10 & 10 & 2 & 0.35 & 0.34 & 0.42 & 0.41 & $\ldots$ & 0.40 \\
\hline & 10 & 10 & 3 & 0.35 & 0.34 & 0.42 & 0.41 & 0.67 & 0.40 \\
\hline & 10 & 10 & 10 & 0.35 & 0.34 & 0.43 & 0.42 & 0.43 & 0.42 \\
\hline \multirow[t]{3}{*}{+1000} & 1 & 0 & 0 & 0.36 & 0.36 & 0.43 & 0.42 & 0.44 & 0.43 \\
\hline & 10 & 1 & 0 & 0.36 & 0.36 & 0.45 & 0.45 & 0.45 & 0.45 \\
\hline & 10 & 10 & 10 & 0.36 & 0.36 & 0.45 & 0.45 & 0.46 & 0.46 \\
\hline
\end{tabular}


Table 4. Correlation of milk-yield lactation daughter yield-deviations with EBV by average number of testday records of daughters for Black and White Holstein bulls that had at least 10 daughters with lactation passed 120 DIM and in at least 10 herds in a May 2003 genetic evaluation.

\begin{tabular}{|c|c|c|c|c|c|c|}
\hline \multirow{2}{*}{$\begin{array}{l}\text { Average no. of } \\
\text { tests of daughters }\end{array}$} & \multicolumn{2}{|c|}{ Lactation 1} & \multicolumn{2}{|c|}{ Lactation 2} & \multicolumn{2}{|c|}{ Lactation 3} \\
\hline & No. of bulls & Correlation & No. of bulls & Correlation & No. of bulls & Correlation \\
\hline 3 & 171 & 0.943 & 88 & 0.901 & 38 & 0.903 \\
\hline 4 & 217 & 0.983 & 208 & 0.976 & 197 & 0.971 \\
\hline 5 & 233 & 0.997 & 338 & 0.993 & 291 & 0.989 \\
\hline 6 & 368 & 0.996 & 390 & 0.996 & 512 & 0.996 \\
\hline 7 & 1210 & 0.997 & 1266 & 0.998 & 2080 & 0.997 \\
\hline 8 & 7131 & 0.998 & 6220 & 0.998 & 4604 & 0.997 \\
\hline 9 & 2111 & 0.998 & 1966 & 0.997 & 1131 & 0.997 \\
\hline 10 & 35 & 0.993 & 41 & 0.997 & 17 & 0.987 \\
\hline
\end{tabular}

should also be applicable for other livestock species, e.g., swine or beef cattle.

\section{Calculation of DYD and Associated Reliabilities}

Formula [19] for calculating DYD and formula [21] for approximating associated reliability are valid for general multiple-trait models, including RRTDM. Calculation of DYD using formula [19] requires estimates of all fixed effects and nongenetic random effects and EBV of mates of bulls from a genetic evaluation. The size of contemporary groups and absorption of nongenetic random effects are considered in computing data contribution of daughters in reliability calculations, and reliability values of mates of a bull are adjusted for as well. For the calculation of DYD of a bull, only records of his own daughters have to be considered; male progeny must be excluded because they do not have their own performance records for production traits. The contribution of granddaughters of the bull to his DYD must be ignored, otherwise the contribution would be double counted, as the granddaughters would contribute their data to the DYD of his sons. In DYD calculation, only the cow-to-sire path is considered. Other paths, such as son to sire, are ignored. This is because the contribution of male progeny to the bull will be accounted for in subsequent genetic analyses, e.g., international bull evaluations (Fikse and Banos, 2001), marker-assisted genetic evaluation (Szyda et al., 2002), and 2-step multiple-trait evaluations based on evaluation results from single-trait models (Ducrocq et al., 2001). Because the DYD of one bull do not affect the DYD of other bulls, DYD calculation can be done on a within-bull basis. Parental contribution to the bull is irrelevant for the calculation of the DYD of bulls.

For genetic analyses, DYD on a 305-d lactation basis are more useful than the DYD vector q expressed on a daily basis in the form of regression coefficients; therefore, DYD on a daily basis need to be converted to 305-d lactation basis. Due to the mathematical function for modeling genetic effects in RRTDM, lactation DYD cannot always be estimated. In case that there are no data available in some lactations or some lactation DYD are not estimable, only a submatrix of matrix B needs to be inverted, and the other parts of the

Table 5. Correlation of milk-yield lactation daughter yield-deviations with EBV by number of daughters for Black and White Holstein bulls that had at least 10 daughters with lactation passed 120 DIM and in at least 10 herds in a May 2003 genetic evaluation.

\begin{tabular}{|c|c|c|c|c|c|c|}
\hline \multirow{2}{*}{$\begin{array}{l}\text { No. of } \\
\text { daughters }\end{array}$} & \multicolumn{2}{|c|}{ Lactation 1} & \multicolumn{2}{|c|}{ Lactation 2} & \multicolumn{2}{|c|}{ Lactation 3} \\
\hline & No. of bulls & Correlation & No. of bulls & Correlation & No. of bulls & Correlation \\
\hline $10-19$ & 446 & 0.989 & 600 & 0.988 & 859 & 0.992 \\
\hline $20-29$ & 545 & 0.994 & 870 & 0.994 & 1216 & 0.994 \\
\hline $30-39$ & 528 & 0.996 & 824 & 0.994 & 1211 & 0.995 \\
\hline $40-49$ & 472 & 0.992 & 789 & 0.994 & 1105 & 0.996 \\
\hline $50-59$ & 409 & 0.994 & 967 & 0.995 & 976 & 0.996 \\
\hline $60-69$ & 523 & 0.991 & 1026 & 0.996 & 726 & 0.996 \\
\hline $70-79$ & 750 & 0.993 & 979 & 0.996 & 566 & 0.997 \\
\hline $80-89$ & 958 & 0.996 & 837 & 0.997 & 395 & 0.998 \\
\hline $90-99$ & 986 & 0.997 & 625 & 0.998 & 303 & 0.998 \\
\hline 100-199 & 4182 & 0.998 & 1936 & 0.998 & 785 & 0.998 \\
\hline $200-499$ & 847 & 0.998 & 473 & 0.998 & 344 & 0.998 \\
\hline$>499$ & 844 & 0.998 & 596 & 0.998 & 385 & 0.998 \\
\hline
\end{tabular}


Table 6. Correlation of milk-yield lactation daughter yield-deviations with EBV by birth year for Black and White Holstein bulls that had at least 10 daughters with lactation passed 120 DIM and in at least 10 herds in a May 2003 genetic evaluation.

\begin{tabular}{|c|c|c|c|c|c|c|}
\hline \multirow[b]{2}{*}{ Birth year } & \multicolumn{2}{|c|}{ Lactation 1} & \multicolumn{2}{|c|}{ Lactation 2} & \multicolumn{2}{|c|}{ Lactation 3} \\
\hline & No. of bulls & Correlation & No. of bulls & Correlation & No. of bulls & Correlation \\
\hline 1986 & 746 & 0.996 & 1122 & 0.996 & 1094 & 0.995 \\
\hline 1987 & 1021 & 0.996 & 1010 & 0.996 & 960 & 0.995 \\
\hline 1988 & 1063 & 0.997 & 1053 & 0.997 & 1013 & 0.996 \\
\hline 1989 & 775 & 0.997 & 759 & 0.997 & 720 & 0.997 \\
\hline 1990 & 808 & 0.997 & 790 & 0.997 & 755 & 0.997 \\
\hline 1991 & 835 & 0.998 & 818 & 0.996 & 783 & 0.994 \\
\hline 1992 & 888 & 0.997 & 863 & 0.996 & 814 & 0.995 \\
\hline 1993 & 945 & 0.996 & 904 & 0.994 & 874 & 0.995 \\
\hline 1994 & 970 & 0.994 & 933 & 0.996 & 910 & 0.995 \\
\hline 1995 & 1039 & 0.997 & 1025 & 0.996 & 863 & 0.987 \\
\hline 1996 & 1075 & 0.998 & 1001 & 0.992 & 85 & 0.953 \\
\hline 1997 & 1036 & 0.990 & 244 & 0.941 & & \\
\hline 1998 & 289 & 0.960 & & & & \\
\hline All & 11,490 & 0.996 & 10,522 & 0.996 & 8871 & 0.996 \\
\hline
\end{tabular}

inverse of matrix $\mathbf{B}$ are set to zero for obtaining a generalized inverse of $\mathbf{B}$. Lactation DYD estimated from shorter lactations are more influenced by extrapolation than lactation DYD from longer lactations. To minimize the impact of extrapolation, at least 10 daughters are required to pass 120 DIM in a lactation in order to make DYD of this lactation official for a bull, which was determined in an application study using the data from a May 2003 evaluation. Similarly, lactation-yield deviations for a lactation of a cow will be publishable if her lactation passes 210 DIM or she has at least 7 tests. Computer resources required for the calculation of the DYD of bulls and lactation-yield deviations of cows and their associated reliabilities were rather limited. Total CPU was equivalent to 8 rounds of iteration for solving mixed-model equations (Liu et al., 2001) for May 2003 production trait genetic evaluations.

The verification study was conducted using Maple 6 software. Estimated breeding value and reliabilities of the bull obtained from the DYD-based mixed-model equation system were identical to those from the complete mixed-model equation system in all scenarios. The reliability values calculated using the MTEDC were equal to their true values. EBV of the daughters obtained from the 2 solving procedures for the complete mixed model equation system were also equal. The results proved that the DYD formula [19] and the reliability formula [21] are correct. A similar verification study was done for a repeatability model, and the DYD and associated reliability formulas were confirmed to be correct for the single-trait model as well. Table 3 shows lactation DYD and EBV of the simulated bull during the course of lactation of his daughters. When all daughters had only 2 test-day records in a lactation, which was smaller than the order of fit for genetic effects, DYD of the bull could not be estimated for the lactation. Even in this case, EBV of the bull could be estimated because left-hand side and righthand side of the DYD-based mixed-model equation system existed in spite of the inestimable DYD. For short lactations, DYD were greater than EBV, with the exception that both were nearly equal in cases of at least 100 daughters. As lactation progressed, the difference between DYD and EBV became smaller, and this was more evident with higher numbers of daughters. The DYD of missing lactations were zero, but corresponding EBV were not. Adding 1000 lactations with 1 test each from second-crop daughters caused changes in both DYD and EBV for the bull. However, these changes were much smaller than those caused by short lactations of first-crop daughters.

It can be seen in Table 4 that, as lactations of daughters became longer or more complete, correlation of lactation DYD with EBV of bulls increased significantly. When the average number of test-day records of daughters reached 5 , the correlation was stabilized. The DYD and EBV of later lactations were less correlated than first lactations, in particular when daughters had, on average, only 3 tests in a lactation. This can be explained by the fact that later lactation EBV were estimated from more sources of information, including daughter information of earlier lactations, whereas first lactation EBV were influenced predominantly by first-lactation daughter information and parental average only. Table 5 indicates that correlation between lactation DYD and EBV increased slightly with the number of daughters. In Table 6 , correlations of lactation DYD with EBV were close to unity, except for the very last birth year, where bulls tended to have fewer daughters and higher percentages of daughters with less completed lactations. Due to shorter lacta- 
tions of second-crop daughters, bulls born in 1994, 1993 , and 1991 to 1992 , had slightly lower correlations for first, second, and third lactations, respectively. Across all birth years of bulls, correlation between lactation DYD and EBV was 0.996 for all 3 lactations. Summarizing the correlations in Tables 4, 5, and 6, we concluded that the DYD formula [19] is accurate.

Because the DYD formula [19] expresses DYD in the form of regression coefficients for RRTDM, any linear function of the regression coefficient estimates can be derived for individual bulls. For routine genetic evaluations, 305-d lactation DYD values and DYD lactation curves for bulls satisfying the requirement for official DYD are published, in addition to lactation EBV and genetic lactation curves.

\section{CONCLUSIONS}

A multiple lactation RRTDM is used for genetic evaluations of milk, fat, protein yield, or SCS for joint analyses of Holstein, Red, and Jersey dairy cattle from Austria, Germany, and Luxembourg. For reliability approximation, the MTEDC was feasible for multitrait models, reasonably accurate, and applicable for very large populations (e.g., a German Holstein population with more than 11 million cows and 15 million animals). The presented DYD formula for multitrait models was verified to be correct in a simulation study. The correlations between DYD and EBV from a routine genetic evaluation were very high-0.996 for all bullssuggesting that the DYD were highly consistent with EBV. The developed DYD and MTEDC methods can be used to compute DYD and associated reliabilities for international bull comparisons based on a multipletrait model, a marker-assisted genetic evaluation, and validation of a national genetic evaluation system.

\section{REFERENCES}

Biochard, D., B. Bonaiti, A. Barbat, and S. Mattalia. 1995. Three methods to validate the estimation of genetic trend for dairy cattle. J. Dairy Sci. 78:431-437.

Fikse, W. F., and G. Banos. 2001. Weighting factors of sire daughter information in international genetic evaluations. J. Dairy Sci. 84:1759-1767.

De Roos, A. P. W., A. G. F. Harbers, and G. de Jong. 2002. Herd specific random regression curves in a test-day model for protein yield in dairy cattle. Pages 55-58 in Proc. 7 WCGALP, Communication \#01-05, Montpellier, France.

Ducrocq, V., D. Boichard, A. Barbat, and H. Larroque. 2001. Implementation of an approximate multi-trait BLUP evaluation to combine production traits and functional traits into a total merit index. Page 2 in 52nd Annual Meeting of the European Association for Animal Production, Budapest, Hungary.

Graser, H. U., and B. Tier. 1997. Applying the concept of number of effective progeny to approximate accuracies of predictions derived from multiple-trait analyses. Pages 547-551 in Proc. Assoc. Adv. Anim. Breed. Genet., Dubbo, NSW, Australia.
Haile-Mariam, M., M. E. Goddard, and P. J. Bowman. 2001. Estimates of genetic parameters for daily somatic cell count of Australian dairy cattle. J. Dairy Sci. 84:1255-1264.

Harris, B., and D. Johnson. 1998. Approximate reliabilities of genetic evaluations under an animal model. J. Dairy Sci. 81:2723-2728.

Harville, D. A. 1997. Matrix Algebra from a Statistician's Perspective. Springer, New York, NY.

Henderson, C. R. 1984. Applications of Linear Models in Animal Breeding. University of Guelph, Guelph, Ontario, Canada.

Jamrozik, J., L. R. Schaeffer, and G. B. Jansen. 2000. Approximate accuracies of prediction from random regression models. Livest. Prod. Sci. 66:85-92.

Koots, K. R., L. R. Schaeffer, and G. B. Jansen. 1997. Approximate accuracy of genetic evaluation under an animal model. J. Dairy Sci. 80 (Suppl. 1):226. (Abstr.)

Liu, Z., F. Reinhardt, and R. Reents. 2000a. Estimating parameters of a random regression test day model for first three lactation milk production traits using the covariance function approach. Interbull Bull. 25:74-80.

Liu, Z., F. Reinhardt, and R. Reents. 2000b. Parameter estimates of a random regression test day model for first three lactation somatic cell scores. Interbull Bull. 26:61-66.

Liu, Z., F. Reinhardt, A. Bünger, L. Dopp, and R. Reents. 2001. Application of a random regression model to genetic evaluations of test day yields and somatic cell scores in dairy cattle. Interbull Bull. 27:159-166.

Liu, Z., F. Reinhardt, and R. Reents. 2002. The multiple trait effective daughter contribution method applied to approximate reliabilities of estimated breeding values of a random regression test day model for genetic evaluation in dairy cattle. Pages 553556 in Proc. 7WCGALP, Communication \#20-15, Montpellier, France.

Mrode, R. A. 1996. Linear models for the prediction of animal breeding values. CAB International, Wallingford, UK.

Mrode, R. A., and G. J. T. Swanson. 2002. The calculation of cow and daughter yield deviations and partitioning of genetic evaluations when using a random regression model. Pages 51-54 in Proc. 7 WCGALP, Communication \#01-04, Montpellier, France.

Reents, R., J. C. M. Dekkers, and L. R. Schaeffer. 1995. Genetic evaluation for somatic cell score with a test day model for multiple lactations. J. Dairy Sci. 78:2858-2870.

Schaeffer, L. R., J. Jamrozik, G. J. Kistemaker, and B. J. Van Doormaal. 2000. Experience with a test day model. J. Dairy Sci. 83:1135-1144.

Schaeffer, L. R. 2001. Multiple trait international bull comparison. Livest. Prod. Sci. 69:145-153.

Strabel, T., I. Misztal, and J. K. Bertrand. 2001. Approximation of reliabilities for multiple-trait model with maternal effects. J. Anim. Sci. 79:833-839.

Strandén, I., and M. Lidauer. 1999. Solving large mixed linear models using preconditioned conjugate gradient iteration. J. Dairy Sci. 82:2779-2787.

Szyda, J., Z. Liu, R. Maschka, F. Reinhardt, and R. Reents. 2002. Computer system for routine QTL detection and genetic evaluation under a mixed inheritance model in dairy cattle. Pages 749750 in Proc. 7 WCGALP, Communication \#28-10, Montpellier, France.

VanRaden, P. M. 2001. Methods to combine estimated breeding values obtained from separate source. J. Dairy Sci. 84 (E. Suppl.):E47-E55.

VanRaden, P. M., and G. R. Wiggans. 1991. Derivation, calculation, and use of national animal model information. J. Dairy Sci. 74:2737-2746.

Wiggans, G. R., P. M. VanRaden, J. Bormann, J. C. Philpot, T. Druet, and N. Gengler. 2002. Deriving lactation yields from testday yields adjusted for lactation stage, age, pregnancy, and herd test date. J. Dairy Sci. 85:264 (online only). http://www.adsa.org/ jds/abs/2002/d0210264.htm. 


\section{APPENDIX 1}

\section{Derivation of the Formula for Computing Animal's Reliability Contributed by a Progeny Adjusted for Mate of the Animal}

Formula [13] is used for calculating an animal's reliability contributed by a progeny adjusted for mate of the animal. To derive the formula, we apply here a similar derivation method for general multiple-trait models as VanRaden and Wiggans (1991) did for a single-trait repeatability animal model. Let $\mathbf{y}^{*}$ represent a vector of adjusted trait values of a progeny, from which true values, instead of estimates, of all fixed and nongenetic random effects have been subtracted. Let $\mathbf{y}^{o}$ be $\mathbf{y}^{*}$ adjusted for the effect of mate of the animal $\left(\hat{\mathbf{a}}_{d}\right)$

$$
\mathbf{y}^{o}=\mathbf{y}^{*}-\frac{1}{2} \hat{\mathbf{a}}_{d}
$$

A sire model can be used to predict the animal's breeding values based on this progeny's information only

$$
\mathbf{y}^{o}=\mathbf{Z} \mathbf{a}_{s}+\omega
$$

with $\mathbf{a}_{s}$ being a vector of the animal's breeding values as sire of the progeny, and $\omega$ is a vector of residual effects containing the progeny's Mendelian sampling and error effects.

If $\mathbf{y}^{*}$ is the only source of information of the progeny for estimating its breeding values $\left(\mathbf{a}_{\mathrm{P}}\right)$, then the corresponding mixed-model equations would be

$$
\left(\mathbf{Z}^{\prime} \mathbf{R}^{-1} \mathbf{Z}+\mathbf{G}_{0}^{-1}\right) \hat{\mathbf{a}}_{\mathrm{P}}=\mathbf{Z}^{\prime} \mathbf{R}^{-1} \mathbf{y}^{*} .
$$

Reliability of the progeny contributed by its own data only is computed with

$$
\begin{aligned}
\Re_{\mathrm{P}}^{*} & =\mathbf{I}-\mathbf{G}_{0}^{-1}\left(\mathbf{Z}^{\prime} \mathbf{R}^{-1} \mathbf{Z}+\mathbf{G}_{0}^{-1}\right)^{-1} \\
& =\mathbf{I}-\left(\mathbf{Z}^{\prime} \mathbf{R}^{-1} \mathbf{Z G}_{0}+\mathbf{I}\right)^{-1}
\end{aligned}
$$

As a result of adjusting for the mate's EBV, genetic variance of $\mathbf{y}^{o}$ is reduced to

$$
\mathbf{G}_{0}^{o}=\mathbf{G}_{0}\left(\mathbf{I}-\frac{1}{4} \Re_{\mathrm{M}}^{*}\right)
$$

The following equations are required in the derivation

$$
\begin{gathered}
\mathbf{Z}^{\prime} \mathbf{R}^{-1} \mathbf{Z}=\left[\left(\mathbf{I}-\Re_{\mathrm{P}}^{*}\right)^{-1}-\mathbf{I}\right] \mathbf{G}_{0}^{-1}, \mathbf{Z}^{\prime} \mathbf{R}^{-1} \mathbf{Z G}_{0} \\
=\left(\mathbf{I}-\Re_{\mathrm{P}}^{*}\right)^{-1}-\mathbf{I} \\
\text { and }\left(\mathbf{Z}^{\prime} \mathbf{R}^{-1}+\left(\mathbf{G}_{0}^{o}\right)^{-1}\right)^{-1}
\end{gathered}
$$

$$
=\mathbf{G}_{0}\left[\left(\mathbf{I}-\mathfrak{R}_{\mathrm{P}}^{*}\right)^{-1}+\left(\mathbf{I}-\frac{1}{4} \Re_{\mathrm{M}}^{*}\right)^{-1}-\mathbf{I}\right]^{-1}
$$

The following (co)variances are also used in the derivation: $\operatorname{var}\left(\mathbf{y}^{o}\right)=\mathbf{V}_{o}=\mathbf{R}+\mathbf{Z G}_{0}^{o} \mathbf{Z}^{\prime}$ and $\operatorname{cov}\left(\mathbf{a}_{s}, \mathbf{y}^{o}\right)=\mathbf{Z G}_{s}$ where $\mathbf{G}_{s}=\frac{1}{4} \mathbf{G}_{0}$. Because

$$
\mathbf{V}_{o}^{-1}=\left(\mathbf{R}+\mathbf{Z G}_{0}^{o} \mathbf{Z}^{\prime}\right)^{-1}=\mathbf{R}^{-1}
$$

$$
\begin{gathered}
-\mathbf{R}^{-1} \mathbf{Z}\left(\mathbf{Z}^{\prime} \mathbf{R}^{-1} \mathbf{Z}+\left(\mathbf{G}_{0}^{o}\right)^{-1}\right)^{-1} \mathbf{Z}^{\prime} \mathbf{R}^{-1}(\text { Harville, } 1997) \\
\mathbf{Z}^{\prime} \mathbf{V}_{o}^{-1} \mathbf{Z} \mathbf{G}_{0}=\mathbf{Z}^{\prime} \mathbf{R}^{-1} \mathbf{Z} \mathbf{G}_{0} \\
-\mathbf{Z}^{\prime} \mathbf{R}^{-1} \mathbf{Z}\left(\mathbf{Z}^{\prime} \mathbf{R}^{-1} \mathbf{Z}+\left(\mathbf{G}_{0}^{o}\right)^{-1}\right)^{-1} \mathbf{Z}^{\prime} \mathbf{R}^{-1} \mathbf{Z G}_{0}
\end{gathered}
$$

An animal's breeding values, based only on the information of this progeny, are estimated with (Henderson, 1984, p. 39)

$$
\hat{\mathbf{a}}_{s}=\mathbf{G}_{s} \mathbf{Z}^{\prime} \mathbf{V}_{o}^{-1} \mathbf{y}^{o}
$$

Because $\operatorname{var}\left(\hat{\mathbf{a}}_{s}\right)=\mathbf{G}_{s} \mathbf{Z}^{\prime} \mathbf{V}_{o}^{-1} \mathbf{Z} \mathbf{G}_{s}$, and animals's reliability contributed by the progeny and adjusted for the mate of the animal is

$$
\Re_{\mathrm{P}-\mathrm{M}}=\mathbf{G}^{-1}{ }_{s} \mathbf{G}_{s} \mathbf{Z}^{\prime} \mathbf{V}_{o}^{-1} \mathbf{Z} \mathbf{G}_{s}=\frac{1}{4} \mathbf{Z}^{\prime} \mathbf{V}_{o}^{-1} \mathbf{Z} \mathbf{G}_{0}
$$

$$
\begin{gathered}
\text { Let } \mathbf{E}=\mathbf{Z}^{\prime} \mathbf{R}^{-1} \mathbf{Z} \mathbf{G}_{0}=\left(\mathbf{I}-\Re_{\mathrm{P}}^{*}\right)^{-1}-\mathbf{I} \text {, then } \Re_{\mathrm{P}-\mathrm{M}} \\
=\frac{1}{4}\left[\mathbf{Z}^{\prime} \mathbf{R}^{-1} \mathbf{Z G}_{0}-\mathbf{Z}^{\prime} \mathbf{R}^{-1} \mathbf{Z}\left(\mathbf{Z}^{\prime} \mathbf{R}^{-1} \mathbf{Z}+\left(\mathbf{G}_{0}^{o}\right)^{-1}\right)^{-1} \mathbf{Z}^{\prime} \mathbf{R}^{-1} \mathbf{Z G}_{0}\right] \quad[\mathrm{A} 8] \\
=\frac{1}{4}\left[\mathbf{E}-\left[\left(\mathbf{I}-\Re_{\mathrm{P}}^{*}\right)^{-1}-\mathbf{I}\right]\left[\left(\mathbf{I}-\Re_{\mathrm{P}}^{*}\right)^{-1}+\left(\mathbf{I}-\frac{1}{4} \Re_{\mathrm{M}}^{*}\right)^{-1}-\mathbf{I}\right]^{-1} \mathbf{E}\right] \\
=\frac{1}{4}\left[\mathbf{E}-\mathbf{E}\left(\mathbf{E}+\left(\mathbf{I}-\frac{1}{4} \Re_{\mathrm{M}}^{*}\right)^{-1}\right)^{-1} \mathbf{E}\right]
\end{gathered}
$$

The above formula [A8] was derived for progeny with performance records. In case that progeny has no performance data, $\mathbf{Z}^{\prime} \mathbf{R}^{-1} \mathbf{Z}$ is replaced with an EDC matrix. Whether an EDC matrix or $\mathbf{Z}^{\prime} \mathbf{R}^{-1} \mathbf{Z}$ are considered, the same formula for $\Re_{\mathrm{P}-\mathrm{M}}$ can be obtained.

\section{APPENDIX 2}

\section{Derivation of the Formula for Computing Daughter Yield Deviations of Bulls under Multiple Trait Models}

Formula [19] is used for computing DYD of bulls under general multiple-trait models, including RRTDM. To derive the DYD formula for multiple-trait models, we apply a similar approach here as used by VanRaden 
and Wiggans (1991) for a single-trait model. Assuming a cow $i$ has its own performance records and no progeny, equations of genetic effects for the cow i can be written in a general way as follows

$$
\begin{gathered}
\mathbf{Z}_{i}^{\prime} \mathbf{R}_{i}^{-1} \mathbf{X}_{i} \hat{\mathbf{b}}+\mathbf{Z}_{i}^{\prime} \mathbf{R}_{i}^{-1} \mathbf{Z}_{p i} \hat{\mathbf{p}}_{i} \\
+\left(\mathbf{Z}_{i}^{\prime} \mathbf{R}_{i}^{-1} \mathbf{Z}_{i}+a_{*}^{i i} \mathbf{G}_{0}^{-1}\right) \tilde{\mathbf{a}}_{i} \\
-\frac{1}{2} d_{i} \mathbf{G}_{0}^{-1}\left(\tilde{\mathbf{a}}_{s}+\hat{\mathbf{a}}_{d}\right)=\mathbf{Z}_{i}^{\prime} \mathbf{R}_{i}^{-1} \mathbf{y}_{i}
\end{gathered}
$$

where EBV of the dam of the cow $\left(\hat{\mathbf{a}}_{d}\right)$ are obtained from a complete evaluation system, e.g., from a national genetic evaluation. $\tilde{\mathbf{a}}_{i}$ is EBV of the cow $\mathrm{i}$ that were estimated as if only her own performance and her parents made a contribution to her EBV. $\tilde{\mathbf{a}}_{s}$ is the EBV of the sire of the cow $i$ that were estimated as if only his daughters contributed to his EBV. For computing the contribution of the cow to her sire's DYD, only her performance records are considered, and the contribution of her progeny to her EBV must be ignored because her daughters' records make a contribution to her mates' DYD and her granddaughters to her sons. $d_{i}$ is the diagonal element pertinent to cow $\mathrm{i}$ in inverse of the (co)variance matrix of Mendelian sampling $\mathbf{D}^{-1}$ (Mrode, 1996). Without its own progeny, $d_{i}=a_{*}^{i i}$. Note that the sire and his mate are allowed to be inbred. Rewriting equation [B1] gives EBV of the cow based on her records and parental information

$$
\tilde{\mathbf{a}}_{i}=\mathbf{Q}_{i} \mathbf{Z}_{i}^{\prime} \mathbf{R}_{i}^{-1} \varepsilon_{i}+\frac{1}{2} d_{i} \mathbf{Q}_{i} \mathbf{G}_{0}^{-1}\left(\tilde{\mathbf{a}}_{s}+\hat{\mathbf{a}}_{d}\right)
$$

where $\varepsilon_{i}=\mathbf{y}_{i}-\mathbf{X}_{i} \hat{\mathbf{b}}-\mathbf{Z}_{p i} \hat{\mathbf{p}}_{i}$ and $\mathbf{Q}_{i}=\left(\mathbf{Z}_{i}^{\prime} \mathbf{R}_{i}^{-1} \mathbf{Z}_{i}+a_{*}^{i i} \mathbf{G}_{0}^{-1}\right)^{-1}$

Define average yield deviations for the cow $i$ as

$$
\mathbf{c}_{i}=\left(\mathbf{Z}_{i}^{\prime} \mathbf{R}_{i}^{-1} \mathbf{Z}_{i}\right)^{-1} \mathbf{Z}_{i}^{\prime} \mathbf{R}_{i}^{-1} \varepsilon_{i}
$$

For the RRTDM, $\mathbf{c}_{i}$ are expressed in the form of regression coefficients. Lactation or average-yield deviations do not exist for lactation of cow $i$ when she has fewer tests in the lactation than the order of fit for genetic effects. Replacing $\varepsilon_{i}$ in equation [B2] with $\mathbf{c}_{i}$ gives

$$
\tilde{\mathbf{a}}_{i}=\mathbf{Q}_{i} \mathbf{Z}_{i}^{\prime} \mathbf{R}_{i}^{-1} \mathbf{Z}_{i} \mathbf{c}_{i}+\frac{1}{2} d_{i} \mathbf{Q}_{i} \mathbf{G}_{0}^{-1}\left(\tilde{\mathbf{a}}_{s}+\hat{\mathbf{a}}_{d}\right)
$$

Because no other sources of information are involved,

$$
\mathbf{Q}_{i} \mathbf{Z}_{i}^{\prime} \mathbf{R}_{i}^{-1} \mathbf{Z}_{i}+d_{i} \mathbf{Q}_{i} \mathbf{G}_{0}^{-1}=\mathbf{I}
$$

Genetic effect equations for a bull without its own performance can be written as

$$
\begin{gathered}
a^{s s} \mathbf{G}_{0}^{-1} \tilde{\mathbf{a}}_{s}=\frac{1}{2} d_{s} \mathbf{G}_{0}^{-1}\left(\hat{\mathbf{a}}_{s s}+\hat{\mathbf{a}}_{d s}\right) \\
+\sum_{i=1}^{n} \frac{1}{2} d_{i} \mathbf{G}_{0}^{-1}\left(\tilde{\mathbf{a}}_{i}-\frac{1}{2} \hat{\mathbf{a}}_{d}\right),
\end{gathered}
$$

where $\tilde{\mathbf{a}}_{s}, \hat{\mathbf{a}}_{s s}$, and $\hat{\mathbf{a}}_{d s}$ represent EBV of the bull, his sire, and dam, respectively. $d_{s}$ is diagonal element of matrix $\mathbf{D}^{-1}$ (Mrode, 1996, p. 28) for the bull. Note that no male progeny of the bull is considered, because his sons do not have their own production records.

$$
a^{s s}=d_{s}+\sum_{i=1}^{n} \frac{1}{4} d_{i}
$$

where $\mathrm{n}$ is the number of daughters with data. Substitution of equation [B4] into equation [B6] by treating the cow $i$ as one of $n$ daughters of the bull gives

$$
\begin{gathered}
a^{s s} \mathbf{G}_{0}^{-1} \tilde{\mathbf{a}}_{s}=\frac{1}{2} d_{s} \mathbf{G}_{0}^{-1}\left(\hat{\mathbf{a}}_{s s}+\hat{\mathbf{a}}_{d s}\right)+\sum_{i=1}^{n} \frac{1}{2} d_{i} \mathbf{G}_{0}^{-1} \\
\left(\mathbf{Q}_{i} \mathbf{Z}_{i}^{\prime} \mathbf{R}_{i}^{-1} \mathbf{Z}_{i} \mathbf{c}_{i}+\frac{1}{2} d_{i} \mathbf{Q}_{i} \mathbf{G}_{0}^{-1}\left(\tilde{\mathbf{a}}_{s}+\hat{\mathbf{a}}_{d}\right)-\frac{1}{2} \hat{\mathbf{a}}_{d}\right) .
\end{gathered}
$$

Accumulating the terms involving $\tilde{\mathbf{a}}_{s}$ to the left side of Equation [B8] leads to

$$
\begin{gathered}
\mathbf{G}\left({ }_{0}^{-1} a^{s s} \mathbf{I}-\sum_{i=1}^{n} \frac{1}{4} d_{i}^{2} \mathbf{Q}_{i} \mathbf{G}_{0}^{-1}\right) \tilde{\mathbf{a}}_{s}=\frac{1}{2} d_{s} \mathbf{G}_{0}^{-1}\left(\hat{\mathbf{a}}_{s s}+\hat{\mathbf{a}}_{d s}\right) \\
+\sum_{i=1}^{n} \frac{1}{2} d_{i} \mathbf{G}_{0}^{-1}\left[\mathbf{Q}_{i} \mathbf{Z}_{i}^{\prime} \mathbf{R}_{i}^{-1} \mathbf{Z}_{i} \mathbf{c}_{i}-\left(\mathbf{I}-d_{i} \mathbf{Q}_{i} \mathbf{G}_{0}^{-1}\right) \frac{1}{2} \hat{\mathbf{a}}_{d}\right]
\end{gathered}
$$

Based on equation [B5], the right side of equation [B9] can be simplified to

$\frac{1}{2} d_{s} \mathbf{G}_{0}^{-1}\left(\hat{\mathbf{a}}_{s s}+\hat{\mathbf{a}}_{d s}\right)+\sum_{i=1}^{n} \frac{1}{2} d_{i} \mathbf{G}_{0}^{-1} \mathbf{Q}_{i} \mathbf{Z}_{i}^{\prime} \mathbf{R}_{i}^{-1} \mathbf{Z}_{i}\left(\mathbf{c}_{i}-\frac{1}{2} \hat{\mathbf{a}}_{d}\right)$

$$
\text { Let } \mathbf{B}=\sum_{i=1}^{n} \frac{1}{4} d_{i} \mathbf{G}_{0}^{-1}\left(\mathbf{I}-d_{i} \mathbf{Q}_{i} \mathbf{G}_{0}^{-1}\right)=\sum_{i=1}^{n} \frac{1}{4} d_{i} \mathbf{G}_{0}^{-1} \mathbf{Q}_{i} \mathbf{Z}_{i}^{\prime} \mathbf{R}_{i}^{-1} \mathbf{Z}_{i}
$$

and

$$
\begin{gathered}
\xi=\sum_{i=1}^{n} \frac{1}{2} d_{i} \mathbf{G}_{0}^{-1} \mathbf{Q}_{i} \mathbf{Z}_{i}^{\prime} \mathbf{R}_{i}^{-1} \mathbf{Z}_{i}\left(\mathbf{c}_{i}-\frac{1}{2} \hat{\mathbf{a}}_{d}\right) \\
=\sum_{i=1}^{n} \frac{1}{2} d_{i} \mathbf{G}_{0}^{-1} \mathbf{Q}_{i} \mathbf{Z}_{i}^{\prime} \mathbf{R}_{i}^{-1}\left(\mathbf{y}_{i}-\mathbf{X}_{i} \hat{\mathbf{b}}-\mathbf{Z}_{p i} \hat{\mathbf{p}}_{i}-\frac{1}{2} \mathbf{Z}_{i} \hat{\mathbf{a}}_{d}\right),
\end{gathered}
$$

The DYD formula for general multiple-trait models is obtained as

$$
\begin{gathered}
\mathbf{q}=\mathbf{B}^{-1} \xi=2\left(\sum_{i=1}^{n} d_{i} \mathbf{G}_{0}^{-1} \mathbf{Q}_{i} \mathbf{Z}_{i}^{\prime} \mathbf{R}_{i}^{-1} \mathbf{Z}_{i}\right)^{-1} \\
\sum_{i=1}^{n} d_{i} \mathbf{G}_{0}^{-1} \mathbf{Q}_{i} \mathbf{Z}_{i}^{\prime} \mathbf{R}_{i}^{-1}\left(\mathbf{y}_{i}-\mathbf{X}_{i} \hat{\mathbf{b}}-\mathbf{Z}_{p i} \hat{\mathbf{p}}_{i}-\frac{1}{2} \mathbf{Z}_{i} \hat{\mathbf{a}}_{d}\right)
\end{gathered}
$$

